\title{
SUSTENTABILIDADE - UMA INVESTIGAÇÃO DA ATITUDE E DO COMPORTAMENTO DE ESTUDANTES DE ADMINISTRAÇÃO
}

\author{
SUSTAINABILITY: AN INVESTIGATION OF THE ATTITUDE \\ AND BEHAVIOR OF MANAGEMENT STUDENTS \\ SOSTENIBILIDAD: UNA INVESTIGACIÓN DE LA ACTITUD Y \\ EL COMPORTAMIENTO DE LOS ESTUDIANTES DE \\ ADMINISTRACIÓN
}

\author{
Danielle Nunes Bezerra de Melo \\ Bacharel em Administração - FEAAC/UFC, \\ Fortaleza, Brasil \\ nunes.danny@gmail.com

\section{Cláudia Buhamra Abreu Romero} \\ Doutora em Administração - EAESP/FGV, São \\ Paulo, Brasil \\ Professora Titular - FEAAC/UFC, Fortaleza, \\ Brasil \\ buhamra@ufc.br \\ Hugo Osvaldo Acosta Reinado \\ Doutor em Administração - EAESP/FGV, \\ Fortaleza, Brasil \\ Professor Associado - FEAAC/UFC, Fortaleza, \\ Brasil \\ hugo@ufc.br \\ Cristiane Buhamra Abreu \\ Doutora em Administração - UNIFOR, Fortaleza, \\ Brasil \\ Professora Adjunta - UNIFOR, Fortaleza, Brasil \\ crisabreu@unifor.br
}

\author{
Contextus \\ ISSN 1678-2089 \\ ISSNe 2178-9258 \\ Organização: Comitê Científico Interinstitucional \\ Editor Científico: Diego de Queiroz Machado \\ Editor Executivo: Carlos Daniel Andrade \\ Avaliação: double blind review pelo SEER/OJS \\ Recebido em 04/08/2018 \\ Aceito em 19/08/2018 \\ $2^{a}$ versão aceita em 01/09/2018
}

\section{RESUMO}

A preocupação com o meio ambiente é tema recorrente no cenário mundial, levando a sustentabilidade do planeta ao foco de discussões entre governos, organizações e sociedade. Para atender as novas demandas do mercado, os gestores organizacionais estão sendo desafiados a adotar estratégias corporativas sustentáveis que estimulem o crescimento financeiro das empresas, ao mesmo tempo em que assumem a responsabilidade socioambiental que lhes cabe. Pensando nos futuros gestores, o presente trabalho tem o objetivo de analisar a atitude e o comportamento ambientais de estudantes do curso de bacharelado em Administração. Classificada como quantitativa descritiva, a presente pesquisa, realizada com 269 alunos do curso de Administração da Universidade Federal do Ceará - UFC, sugere que os estudantes possuem atitudes propensas à sustentabilidade, porém os comportamentos não são favoráveis, sendo classificados como menos conscientes quanto à importância das suas ações para a preservação do meio ambiente.

Palavras-chave: Estudantes de Administração; Meio ambiente; Atitude; Comportamento; Sustentabilidade.

\section{ABSTRACT}

Concern about the environment is a recurring theme on the world stage, making the planet's sustainability a focus of discussions among governments, organizations and society. To meet the new demands of the market, organizational managers are being challenged to adopt sustainable corporate strategies that stimulate the 
financial growth of companies, while assuming the socio-environmental responsibility that they are entitled to. Thinking about the future managers, this work aims to analyze the environmental attitude and behavior of students of the undergraduate program in Administration. Classified as descriptive and quantitative, as well as carried out with 269 students of the Administration program of the Federal University of Ceará - UFC, the study suggests the students have attitudes prone to sustainability, but their behaviors are not favorable, being classified as less conscious about the importance of their actions for the environment preservation.

Keywords: Management Students; Environment; Attitude; Behavior; Sustainability.

\section{RESUMEN}

La preocupación por el medio ambiente es tema recurrente en el escenario mundial, llevando la sustentabilidad del planeta al foco de discusiones entre gobiernos, organizaciones y sociedad. Para atender las nuevas demandas del mercado, los gestores organizacionales están siendo desafiados a adoptar estrategias corporativas sostenibles que estimulen el crecimiento financiero de las empresas, al mismo tiempo que asumen la responsabilidad socioambiental que les corresponde. Pensando en los futuros gestores, el presente trabajo tiene el objetivo de analizar la actitud y el comportamiento ambiental de estudiantes del curso de Bachillerato en Administración. La investigación, realizada con 269 alumnos de este curso en la Universidad Federal de Ceará - UFC, sugiere que los estudiantes poseen actitudes propensas a la sostenibilidad, pero los comportamientos no son favorables, siendo clasificados como menos conscientes en cuanto a la importancia de sus acciones para la preservación del medio ambiente.

Palabras clave: Estudiantes de Administración; Medio ambiente; Actitud; Comportamiento; Sostenibilidad.

\section{INTRODUÇÃO}

A necessidade de preservar, reutilizar e proteger os recursos naturais está se tornando uma regra na sociedade (OTTMAN, 2012), e a responsabilidade ambiental vem influenciando a realidade dos indivíduos e das organizações que buscam adaptar-se aos novos padrões de vida e consumo. Esforços têm sido cobrados dos gestores organizacionais para desenvolver e adotar estratégias que identifiquem, antecipem e satisfaçam as necessidades dos clientes e da sociedade, de uma forma lucrativa e sustentável (PEATTIE; CHARTER, 2003).

Pressupõe-se que o comportamento ambientalmente correto dos gestores contribui para posicionamentos e estratégias corporativas igualmente sustentáveis, levando as organizações a buscarem um novo perfil de administrador. Com base nessa premissa, as instituições de ensino superior, segundo Jacobi et al. (2011), têm se esforçado para formar estudantes de administração com conhecimentos e habilidades necessários para colocar a sustentabilidade nas suas futuras decisões como gestores. Os mesmos autores também afirmam que, durante a última década, muitos programas de graduação e pós-graduação em negócios têm inserido módulos, cursos e componentes de sustentabilidade nos currículos, porém o pensamento voltado para a hegemonia econômica, tratando o aspecto socioambiental como acessório, permanece muito forte em diversas instituições de ensino superior brasileiras. A formação profissional dos administradores ainda se orienta sobretudo pelos resultados organizacionais em detrimento de aspectos relacionados à sustentabilidade (FREITAS, 2014). 
Um estudo realizado por $\mathrm{Wu}$ et al. (2010) analisaram a estrutura curricular de 642 instituições de ensino superior (IES) ao redor do mundo e constataram que apenas $6 \%$ delas possuíam disciplinas diretamente relacionadas à sustentabilidade. A literatura aponta que a forma como as IES vêm abordando o tema não é suficiente para atender às demandas que mercado, governo e sociedade impõem atualmente. A UNESCO (2005), agência incumbida pela ONU de promover a educação ambiental em todos os níveis, desenvolveu o programa intitulado 2005-2014 - a Década da Educação para o Desenvolvimento Sustentável, visando estimular a mudança de atitudes e comportamentos nas populações. Uma das principais ações desse programa é incluir a sustentabilidade no currículo de instituições de ensino superior.

Até o momento, o ensino de Administração tem enfatizado o papel dos gestores e depreciado outros papéis e responsabilidades que indivíduos e grupos possuem na sociedade. Ora, educar indivíduos além de seus papéis profissionais e gerenciais, dados os desafios que a humanidade vem enfrentando e enfrentará, representa não somente uma carga de responsabilidade para o ensino da Administração no século XXI, mas também um convite para se reinventar (JACOBI et al., 2011).

A formação do administrador não deve, portanto, voltar-se apenas para maior produtividade e lucratividade, mas para a formação de indivíduos críticos e conscientes, que ponderam sobre os impactos sociais e ambientais de suas atitudes e decisões e agem responsavelmente perante os desafios e oportunidades que a sociedade propõe e impõe. É nesse contexto que emerge a pergunta de pesquisa: quais a atitude e o comportamento dos futuros profissionais de Administração em relação às questões de sustentabilidade?

Assim, a presente pesquisa tem por objetivo investigar a atitude e o comportamento pró-ambientais de futuros gestores, hoje estudantes de Administração. Destacam-se como objetivos específicos:

a) investigar a atitude ambiental de estudantes de Administração;

b) investigar o comportamento ambiental de estudantes de Administração;

c) identificar o possível gap existente entre atitudes e comportamentos ambientais; e

d) segmentar os estudantes de Administração quanto ao nível de consciência ambiental.

Para as medidas da atitude, o presente trabalho baseia-se na pesquisa intitulada Cultural Differences in Environmental Knowledge, Attitudes, and Behaviors of Canadian 
Danielle Nunes Bezerra de Melo, Claudia Buhamra Abreu Romero, Hugo Osvaldo Acosta Reinado, Cristiane Buhamra Abreu

Consumers, de LAROCHE et al. (2002). Para as medidas do comportamento, por sua vez, utiliza o teste do consumo consciente, da pesquisa do Instituto Akatu, intitulada Akatu 2012: Rumo à sociedade do bem-estar (AKATU, 2013).

Para alcançar os objetivos propostos, realizou-se uma pesquisa quantitativa e descritiva com estudantes do Curso de Administração da UFC - Universidade Federal do Ceará. A UFC foi considerada a melhor universidade do Norte e Nordeste e a $12^{\mathrm{a}}$ do país pelo RUF - Ranking Universitário Folha de 2017 (RUF, 2017), e seu curso de Administração tem nota 5 no ENADE - Exame Nacional de Desempenho de Estudantes (INEP/MEC, 2017). A UFC foi a segunda instituição de ensino superior mais procurada do Brasil no Sisu - Sistema de Seleção Unificada de 2018, sendo o curso de Administração o mais procurado da Universidade (PORTAL G1-CE, 2018).

Espera-se que os resultados obtidos neste trabalho possam contribuir para discutir a relevância de incluir o tema da sustentabilidade nos currículos dos cursos superiores de Administração.

\section{REFERENCIAL TEÓRICO}

Os primeiros debates ligados ao termo sustentabilidade começaram a ganhar força a partir da década de 1970. Em 1983, a Organização das Nações Unidas (ONU), também temerosa das consequências que o desenvolvimento econômico desenfreado e o crescimento do consumo poderiam acarretar para a sociedade, realizou estudos sobre o desenvolvimento social e o meio ambiente. O Relatório da Comissão Brundtland foi apresentado na Assembleia Geral da ONU em 1987 e chegou ao conceito de desenvolvimento sustentável ainda hoje adotado: aquele "que satisfaz as necessidades presentes, sem comprometer a capacidade das gerações futuras de suprir as suas próprias necessidades" (ONU, 1987).

Em 1994, John Elkington formulou o conceito do Triple Bottom Line ou Tripé da Sustentabilidade (DAHLSTROM, 2011), demonstrando que aspectos econômicos, ambientais e sociais devem ser considerados simultaneamente na busca pela sustentabilidade. Para Charter et al. (2002, p. 10), "sustentabilidade econômica deve ser baseada na sustentabilidade ambiental e social, já que não há economia se não houver um planeta”. 


\subsection{Greenwashing e o marketing verde}

Durante o início da "revolução verde", as organizações estavam mais preocupadas em parecer sustentáveis e comercializar produtos verdes que lhes renderiam frutos financeiros imediatos do que, de fato, investir em estratégias e realizar modificações que visassem à sustentabilidade. Essas ações, conhecidas como greenwashing, incitaram na sociedade uma desconfiança em relação à responsabilidade sustentável das organizações e à veracidade do marketing verde (PEATTIE; CRANE, 2005). Com o tempo, entretanto, as organizações compreenderam que o marketing verde vai muito além da propaganda e da venda de produtos ecológicos. Trata-se da valorização da marca da organização através de ações de responsabilidade social e da sua efetiva contribuição para a preservação do planeta. E diversos autores passaram a dedicar seus trabalhos para a definição e utilização dessa filosofia de atuação do marketing, buscando uma abordagem mais social (CRANE, 2000).

Ottman (2012) ressalta, porém, que é necessário o compromisso de todos os níveis da organização, associando o conceito de sustentabilidade à estratégia central do negócio. Assim, os gestores à frente das empresas devem adotar atitudes e comportamentos coerentes com a responsabilidade ambiental empresarial e tornar o marketing verde o centro de sua estratégia de negócio, abrangendo todas as áreas da organização para que elas funcionem em harmonia, buscando a sustentabilidade e gerando valor superior para os clientes. Segundo Daft (2010), a liderança tem a capacidade de influenciar as pessoas para alcançar metas, e "a influência é o efeito que as ações de uma pessoa têm sobre as atitudes, os valores, as crenças ou os comportamentos dos outros" (DAFT, 2010, p. 706).

Para obter sucesso com uma estratégia de marketing voltada para a sustentabilidade, as organizações devem adotar mais do que um discurso sustentável, mas práticas sustentáveis que englobem todos os estágios de produção, tais como a utilização de indicadores de sustentabilidade como ferramenta de gestão, a preocupação com a escolha de matérias-primas renováveis, investimento no uso de alternativas produtivas menos poluentes, elaboração de embalagens recicláveis e a preocupação com a utilização e o descarte pelo consumidor final. Esse é o conceito do marketing positivo cujas ações criam valor para a empresa, para os clientes e para a sociedade (GOPALDAS, 2015). 
Danielle Nunes Bezerra de Melo, Claudia Buhamra Abreu Romero, Hugo Osvaldo Acosta Reinado, Cristiane Buhamra Abreu

O comprometimento da alta administração é um dos fatores críticos de sucesso para criar e implantar processos sustentáveis dentro das organizações. Nesse sentido, espera-se muito dos futuros gestores (GIOVANNINI; KUGLIANSKAS, 2008). Para garantir o desenvolvimento de estratégias adequadas e a disseminação do conceito de sustentabilidade em todos os níveis da empresa, os administradores à frente da gestão dos negócios devem estar alinhados com atitudes e comportamentos pró-ambientais.

\subsection{Atitudes e comportamentos pró-ambientais}

Para a Teoria da Ação Racional (TAR), de Fishbein e Ajzen (1975), o comportamento é naturalmente gerado da atitude, que, por sua vez, é formada com base: no conhecimento (o que se sabe sobre o tema), na afeição (o que se sente sobre o tema) e na intenção (disposição para adotar comportamentos sobre o tema). Desse modo, o comportamento resulta da atitude e é fruto de escolhas totalmente conscientes e racionais do indivíduo.

Contudo, nem sempre uma boa atitude origina um bom comportamento, podendo existir um lapso entre a forma como o indivíduo pensa e a forma como ele age (MOUTINHO; ROAZZI, 2010). Esse gap entre atitude e comportamento é explicado pela Teoria do Comportamento Planejado (TCP), de Ajzen (1985), uma extensão da TAR (FISHBEIN; AJZEN, 1975). Para Ajzen (1985), embora haja disposição do indivíduo para alguns comportamentos baseados na atitude, seu desempenho poderá depender de fatores não motivacionais, como a disponibilidade de oportunidades e recursos (tempo, dinheiro, habilidades, cooperação de outros). Como exemplo, Romero et al. (2017) explicam que um indivíduo pode ter uma atitude positiva em relação à sustentabilidade (preocupando-se com o meio ambiente), mas não adotar comportamentos sustentáveis (como o hábito da reciclagem) devido à falta de infraestrutura na sua cidade (postos e programas de coleta seletiva).

Laroche et al. (2002) destacam dois fatores de influência na formação da atitude que, consequentemente, influenciarão o comportamento ambientalmente correto: a importância e a inconveniência. Segundo os autores, a importância é quando o indivíduo sente que determinada questão ambiental é importante para si ou para a sociedade. A inconveniência refere-se a quão inconveniente é para o indivíduo comportar-se de maneira sustentável. 
Assim, um indivíduo pode até considerar importante a reciclagem, mas achar inconveniente a separação de lixo em casa.

Mas atitudes e comportamentos em relação ao meio ambiente também variam de acordo com a cultura na qual o indivíduo está inserido (LAROCHE et al., 1996; PAUL; MODI; PATEL, 2016). Na mesma perspectiva, a cultura organizacional pode exercer forte influência na formação da atitude e do comportamento ambientalmente responsável de seus stakeholders (funcionários, clientes, fornecedores), a partir da postura de seus administradores na condução das decisões organizacionais. Por isso, a investigação da atitude e do comportamento de futuros gestores é o foco do presente trabalho, cuja metodologia de pesquisa é detalhada a seguir.

\section{METODOLOGIA}

Para executar este trabalho, foi realizada uma pesquisa quantitativo-descritiva, com os estudantes do curso de Administração da Faculdade de Economia, Administração, Atuária e Contabilidade da Universidade Federal do Ceará, com matrícula ativa no período 2017.1, compreendendo os turnos manhã e noite. A totalidade da população é de 865 alunos, sendo: 444 do turno diurno e 421 do turno noturno. Foram estipulados um erro aceitável de $5 \%$ e o valor de $z_{-} \propto=1,96$, o que leva a uma amostra mínima desejável de 266 respondentes. A presente pesquisa obteve 269 questionários válidos.

O instrumento de coleta de dados está fundamentado em dois questionários de pesquisas: Cultural Differences in Environmental Knowledge, Attitudes, and Behaviors of Canadian Consumers (LAROCHE et al., 2002) para as questões de atitude, e o Teste do Consumo Consciente da Pesquisa Akatu 2012: Rumo à sociedade do bem-estar (AKATU, 2013), para as questões de comportamento. O questionário é composto por 32 questões, divididas em três blocos: o primeiro traz informações demográficas sobre o público pesquisado (questões de 1 a 5); o segundo bloco traz questões sobre atitudes frente às questões de sustentabilidade (questões de 6 a 19), e o terceiro bloco traz as questões de comportamento do Teste de Consumo Consciente da Akatu (questões de 20 a 32). As questões dos blocos 2 (atitude) e 3 (comportamento) estão expostas no Quadro 1: 
Quadro 1 - Medidas de atitude e comportamento

\begin{tabular}{|c|c|}
\hline & $\begin{array}{l}\text { Medidas de atitude } \\
\text { (Adaptado de Laroche et al., 2002) }\end{array}$ \\
\hline $\begin{array}{l}\text { a) Gravidade dos problemas } \\
\text { ambientais }\end{array}$ & $\begin{array}{l}\text { Q6. Em nosso país, há tantas árvores que não precisamos nos preocupar com } \\
\text { reciclagem de papel. } \\
\text { Q7. Em países grandes como o Brasil, qualquer poluição que criamos é } \\
\text { facilmente espalhada (dissipada) e, portanto, não me diz respeito. } \\
\text { Q8. Em países grandes como o Brasil, temos tanta eletricidade que não temos } \\
\text { que nos preocupar com a economia de energia. } \\
\text { Q9. Com tanta água em nosso país, eu não vejo por que as pessoas estão } \\
\text { preocupadas com vazamentos de torneiras e com descargas de vasos sanitários. } \\
\text { Q10. A Terra é um sistema fechado, onde tudo acaba voltando ao normal. } \\
\text { Então eu não vejo necessidade de me preocupar com seu estado atual. }\end{array}$ \\
\hline $\begin{array}{l}\text { b) Importância de ser } \\
\text { ambientalmente correto }\end{array}$ & $\begin{array}{l}\text { Q11. A reciclagem vai reduzir a poluição. } \\
\text { Q12. Reciclagem é importante para preservar os recursos naturais. } \\
\text { Q13. Reciclagem salvará terras que seriam utilizadas como depósitos de lixo. }\end{array}$ \\
\hline $\begin{array}{l}\text { c) Nível de responsabilidade } \\
\text { das corporações }\end{array}$ & $\begin{array}{l}\text { Q14. Empresas de alimentos embalados estão agindo de forma responsável } \\
\text { com o meio ambiente. } \\
\text { Q15. As empresas (fabricantes) de papel estão preocupadas com o ambiente. }\end{array}$ \\
\hline $\begin{array}{l}\text { d) Inconveniência de ser } \\
\text { ambientalmente correto }\end{array}$ & $\begin{array}{l}\text { Q16. Manter pilhas de lixo separadas para reciclagem dá muito trabalho. } \\
\text { Q17. Tentar controlar a poluição dá muito trabalho. Por isso não vale a pena. }\end{array}$ \\
\hline \multirow[t]{2}{*}{ e) Pressão Social } & $\begin{array}{l}\text { Q18. As pressões sociais (vizinhos, familiares, amigos) para o comportamento } \\
\text { ambientalmente correto são muito sentidas no Brasil. } \\
\text { Q19. As pressões legais/normativas para o comportamento ambientalmente } \\
\text { correto são muito sentidas no Brasil. }\end{array}$ \\
\hline & $\begin{array}{l}\text { Medidas de comportamento } \\
\text { (Adaptado de Teste do Consumo Consciente Akatu (2013) }\end{array}$ \\
\hline i) Medidas de economia & $\begin{array}{l}\text { Q20. Costumo fechar a torneira enquanto escovo os dentes. } \\
\text { Q21. Evito deixar lâmpadas acesas em ambientes desocupados. } \\
\text { Q22. Espero os alimentos esfriarem antes de guardar na geladeira. } \\
\text { Q23. Desligo aparelhos eletrônicos quando não os estou usando. }\end{array}$ \\
\hline j) Medidas de planejamento & $\begin{array}{l}\text { Q24. Costumo planejar as compras de alimentos. } \\
\text { Q25. Costumo planejar as compras de roupas. } \\
\text { Q26. Costumo pedir nota fiscal (cupom fiscal) quando vou às compras, mesmo } \\
\text { que o fornecedor não a ofereça espontaneamente. } \\
\text { Q27. Costumo ler atentamente os rótulos antes de decidir por uma compra. }\end{array}$ \\
\hline k) Medidas de reciclagem & $\begin{array}{l}\text { Q28. Quando possível, utilizo também o verso das folhas de papel. } \\
\text { Q29. Em minha casa, separo o lixo para reciclagem. }\end{array}$ \\
\hline $\begin{array}{l}\text { l) Medidas de compra } \\
\text { sustentável }\end{array}$ & $\begin{array}{l}\text { Q30. Procuro passar ao maior número possível de pessoas as informações que } \\
\text { aprendo sobre empresas e produtos. } \\
\text { Q31. Comprei produtos orgânicos nos últimos } 6 \text { meses. } \\
\text { Q32. Comprei produtos feitos com material reciclado nos últimos } 6 \text { meses. }\end{array}$ \\
\hline
\end{tabular}

Fonte: elaboração própria.

Os questionários foram aplicados no período de 27 a 31 de maio de 2017, de duas formas: online, por meio de envio do link do questionário para o público-alvo, utilizando a ferramenta Google Docs; e pessoalmente, com aplicação dos questionários nas salas de aula. 


\section{ANÁLISE E DISCUSSÃO DOS RESULTADOS}

A seguir, são apresentados os resultados da pesquisa e a discussão dos resultados.

\subsection{Análise de confiabilidade - atitudes ambientais}

Primeiramente, verificou-se a confiabilidade dos itens avaliados referente a atitudes ambientais através do alfa de Cronbach (alguns itens tiveram suas escalas invertidas para possibilitar comparações no mesmo sentido que as demais). $\mathrm{O}$ valor resultante para a atitude ambiental foi de 0,601, o que caracteriza uma confiabilidade aceitável. Os 14 itens avaliados para a atitude ambiental foram divididos em 5 escalas, os valores dos alfas de Cronbach para as escalas estão apresentados na Tabela 1. O maior alfa observado foi na importância de ser ambientalmente correto $(\alpha=0,694)$, e o menor alfa observado foi para as questões referentes à inconveniência de ser ambientalmente correto $(\alpha=0,332)$.

Tabela 1 - Alfa de Cronbach das escalas de atitudes ambientais

\begin{tabular}{cc}
\hline Escalas & Alfa de Cronbach \\
\hline Gravidade dos problemas ambientais & 0,553 \\
Importância de ser ambientalmente correto & 0,694 \\
Nível de responsabilidade das corporações & 0,551 \\
Inconveniência de ser ambientalmente correto & 0,332 \\
Pressão social & 0,562 \\
\hline
\end{tabular}

Fonte: elaboração própria.

O item g (Carrega a própria sacola de compras) só tem uma afirmativa (Q23), por isso não foi considerado para o Alfa de Cronbach, e foi excluído.

Como os coeficientes alfas obtidos nas escalas foram baixos, foi realizada uma análise de componentes principais para os itens da escala de atitudes ambientais. Para avaliar a análise de componentes principais foi verificado o coeficiente de Kaiser-Meyer-Olkin (KMO), o qual resultou em 0,67, indicando uma adequação moderada da amostra quanto ao uso da análise fatorial, e o teste de esfericidade de Bartlett, o qual apresentou o valor $\mathrm{p}$ $<0,001$, indicando que o modelo fatorial seria adequado aos dados.

Uma análise fatorial sugeriu que a quantidade de fatores a ser utilizada fosse igual a 4. Baseado na carga dos fatores dos itens, os percentuais de variância explicada pelos 4 fatores assumidos foram de 51,29\%, ou seja, 4 fatores explicam 51,29\%, da variabilidade total. 
Danielle Nunes Bezerra de Melo, Claudia Buhamra Abreu Romero, Hugo Osvaldo Acosta Reinado, Cristiane Buhamra Abreu

Outra medida importante da Análise Fatorial são as comunalidades de cada variável que representam a proporção de variância explicada pelos fatores comuns em uma variável. As comunalidades de cada variável são apresentadas na Tabela 2 e nem todas as comunalidades apresentaram um valor aceitável acima de 0,5 sugerido por Hair et al. (2009), o que não sugere um bom ajuste do modelo nos dados. No entanto, optou-se por prosseguir as análises sem retirar essas variáveis com comunalidade menores que 0,5 pois reduziria muito a quantidade de variáveis. Os maiores valores pertencem à importância de ser ambientalmente correto, indicando que elas são as variáveis de maior importância neste estudo, pois apresentam a maior porção da variância compartilhada com todas as outras variáveis consideradas.

Tabela 2 - Comunalidades referente às questões de atitudes ambientais

\begin{tabular}{cc}
\hline Questões & Comunalidade \\
\hline Q6 & 0,482 \\
Q7 & 0,420 \\
Q8 & 0,397 \\
Q9 & 0,434 \\
Q10 & 0,582 \\
Q11 & $\mathbf{0 , 7 0 7}$ \\
Q12 & $\mathbf{0 , 6 1 8}$ \\
Q13 & $\mathbf{0 , 6 2 1}$ \\
Q14 & 0,472 \\
Q15 & 0,513 \\
Q16 & 0,373 \\
Q17 & 0,501 \\
Q18 & 0,513 \\
Q19 & 0,547 \\
\hline \multicolumn{2}{c}{ Fonte: elaboração própria. }
\end{tabular}

Para os fatores do questionário de atitudes ambientais foram estabelecidos 4 fatores, apresentados na Tabela 3 com a carga dos fatores considerados correspondente a cada questão do questionário completo. O primeiro fator do conjunto de atributos associados a atitudes ambientais de estudantes do curso de Administração UFC é representado pelas escalas de gravidade dos problemas ambientais e importância de ser ambientalmente correto. $\mathrm{O}$ fator 2 ao nível de Responsabilidade das corporações e Pressão Social. O fator 3 explica a Importância de ser ambientalmente correto. O fator 4 explica a gravidade dos problemas ambientais e a inconveniência de ser ambientalmente correto. 
Tabela 3 - Cargas fatoriais do questionário de atitudes ambientais

\begin{tabular}{ccccc}
\hline Questões & Fator 1 & Fator 2 & Fator 3 & Fator 4 \\
\hline Q6 & $\mathbf{0 , 5 4 0}$ & $-0,062$ & 0,269 & $-0,338$ \\
Q7 & $\mathbf{0 , 6 0 0}$ & 0,075 & 0,131 & $-0,192$ \\
Q8 & $\mathbf{0 , 4 6 4}$ & 0,008 & 0,302 & 0,301 \\
Q9 & $\mathbf{0 , 5 0 3}$ & 0,010 & 0,424 & 0,032 \\
Q10 & 0,350 & $-0,017$ & 0,238 & $\mathbf{- 0 , 6 3 4}$ \\
Q11 & $\mathbf{0 , 4 6 3}$ & $-0,413$ & $\mathbf{- 0 , 5 6 4}$ & 0,069 \\
Q12 & $\mathbf{0 , 5 2 4}$ & $-0,359$ & $\mathbf{- 0 , 4 3 8}$ & 0,149 \\
Q13 & $\mathbf{0 , 4 6 7}$ & $-0,331$ & $\mathbf{- 0 , 5 0 5}$ & $-0,192$ \\
Q14 & 0,243 & $\mathbf{0 , 6 2 7}$ & $-0,057$ & $-0,126$ \\
Q15 & 0,336 & $\mathbf{0 , 5 9 9}$ & $-0,192$ & $-0,066$ \\
Q16 & 0,399 & $-0,047$ & 0,262 & $\mathbf{0 , 3 7 7}$ \\
Q17 & 0,446 & $-0,149$ & 0,338 & $\mathbf{0 , 4 0 7}$ \\
Q18 & 0,191 & $\mathbf{0 , 6 3 4}$ & $-0,243$ & 0,126 \\
Q19 & 0,212 & $\mathbf{0 , 6 1 3}$ & $-0,303$ & 0,188 \\
\hline \multicolumn{5}{c}{ Fonte: elaboração própria. }
\end{tabular}

A Análise Fatorial Confirmatória (AFC) foi aplicada ao questionário de 14 itens, com intuito de verificar se a estrutura proposta para avaliar a atitude ambiental dos estudantes apresenta um bom ajuste em relação aos dados, utilizando o método de máxima verossimilhança.

De acordo com Byrne (2016), é necessária a análise de diversos índices de ajustamento, de forma a avaliar a adequabilidade do modelo proposto aos dados da amostra. Para verificar a adequação do modelo para cada questionário, foram analisados cinco índices: $\chi^{\wedge} 2$ (Qui-quadrado), juntamente com os graus de liberdade (gl) e valor p; Comparative Fit Index (CFI); Root-Square Error of approximation (RMSEA) e Standardized Root Mean Square Residual (SRMR). De acordo com os resultados avaliados, o questionário de 14 questões (da 06 a 19) apresentou uma boa adequação, como mostra a Tabela 4.

Tabela 4 - Índices calculados para o modelo das escalas de atitude ambiental

\begin{tabular}{cccccc}
\hline$\chi^{\mathbf{2}}(\mathbf{g l})$ & Valor p & CFI & RMSEA & RMSEA (IC) & SRMR \\
\hline $83,227(67)$ & 0,087 & 0,964 & 0,030 & $(0,000-0,049)$ & 0,042 \\
\hline \multicolumn{6}{c}{ Fonte: elaboração própria. }
\end{tabular}

A construção do modelo se deu em particionar o conjunto de dados de atitudes ambientais em escalas, sendo estas: gravidade dos problemas ambientais explicadas pelas 
Danielle Nunes Bezerra de Melo, Claudia Buhamra Abreu Romero, Hugo Osvaldo Acosta Reinado, Cristiane Buhamra Abreu

afirmações 6,7, 8,9 e 10; importância de ser ambientalmente correto explicadas pelas afirmações 11,12 e 13; Nível de responsabilidade das corporações explicada pelas questões 14 e 15; Inconveniência de ser ambientalmente correto explicada pelas afirmações 16 e 17; Pressão social explicada pelas afirmações 18 e 19. O ajuste do modelo foi realizado com o auxílio computacional do Software R.

Como o teste Qui-quadrado é muito sensível ao tamanho da amostra, é indicado verificar a razão entre o valor obtido pelo Qui-quadrado e os respectivos graus de liberdade, nesse caso o valor resultante é de 1,242 considerado um bom ajuste.

\subsection{Análise de confiabilidade - comportamentos ambientais}

Utilizou-se o alfa de Cronbach para verificar a confiabilidade dos itens avaliados referente ao comportamento ambiental, alguns itens tiveram suas escalas invertidas para que fosse possível compará-las no mesmo sentido que as demais. Para essa escala, o coeficiente alfa de Cronbach apresentou confiabilidade aceitável $($ alfa=0,677) de acordo com o limite estabelecido. Os 11 itens avaliados para o comportamento ambiental (Q20 a Q30) foram divididos em 4 escalas, os valores dos alfas de Cronbach para as escalas estão apresentados na Tabela 5. O maior alfa observado foi nos itens de medidas de planejamento $(\alpha=0,636)$, e o menor alfa observado foi para as questões referente a medidas de economia $(\alpha=0,231)$. A escala de compra sustentável não foi avaliada com o alfa de Cronbach devido haver três questões, em que duas delas (Q31 e Q32) não estão em escala de Likert, impossibilitando associar-se com as questões dessa escala.

Tabela 5 - Alfa de Cronbach das escalas de comportamentos ambientais

\begin{tabular}{cc}
\hline Escalas & Alfa de Cronbach \\
\hline Medidas de economia & 0,382 \\
Medidas de planejamento & 0,636 \\
Medidas de reciclagem & 0,231 \\
\hline
\end{tabular}

Fonte: elaboração própria.

Para a análise fatorial exploratória foi realizada uma análise de componentes principais para os itens da escala de comportamentos ambientais. Para avaliar a análise de componentes principais foi verificado o coeficiente de Kaiser-Meyer-Olkin (KMO), o qual 
resultou em 0,76, indicando uma boa adequação da amostra quanto ao uso da análise fatorial, e o teste de esfericidade de Bartlett, o qual apresentou o valor $\mathrm{p}<0,001$, indicando que o modelo fatorial seria adequado aos dados.

Foi realizada uma análise fatorial que sugeriu que a quantidade de fatores a ser utilizada fosse igual a 4. Baseado na carga dos fatores dos 11 itens, os percentuais de variância explicada pelos 4 fatores assumidos foram de 55,67\%, ou seja, 4 fatores explicam $55,67 \%$, da variabilidade total.

As comunalidades de cada variável são apresentadas na Tabela 6 e, como nas atitudes, nem todas as comunalidades de comportamento apresentaram um valor aceitável acima de 0,5 sugerido por Hair et al. (2009), o que não sugere um bom ajuste do modelo nos nossos dados. No entanto, optou-se também por prosseguir com as análises sem retirar essas variáveis com comunalidades menores que 0,5 pois reduziria muito a quantidade de variáveis.

Os maiores valores pertencem a medidas de economia e a de reciclagem, indicando que elas são as variáveis de maior importância nesse estudo, pois apresentam a maior porção da variância compartilhada com todas as outras variáveis consideradas.

Tabela 6 - Comunalidades referentes às questões de comportamentos ambientais

\begin{tabular}{cc}
\hline Questões & Comunalidade \\
\hline Q26 & 0,462 \\
Q27 & 0,606 \\
Q28 & $\mathbf{0 , 6 4 0}$ \\
Q29 & 0,591 \\
Q30 & 0,621 \\
Q31 & 0,609 \\
Q32 & 0,491 \\
Q33 & 0,478 \\
Q34 & 0,377 \\
Q35 & $\mathbf{0 , 7 1 0}$ \\
Q36 & 0,539 \\
\hline
\end{tabular}

Fonte: elaboração própria.

Para os fatores do questionário de comportamentos ambientais foram estabelecidos 4 fatores, apresentados na Tabela 7 com a carga dos fatores considerados correspondente a cada questão do questionário. Constata-se que o primeiro fator do conjunto de atributos associados a comportamentos ambientais de estudantes do curso de Administração UFC é significativamente representado pelas questões referentes a medidas de economia, medidas de planejamento e compra sustentável. Os resultados sugerem que a questão de medidas de 
Danielle Nunes Bezerra de Melo, Claudia Buhamra Abreu Romero, Hugo Osvaldo Acosta Reinado, Cristiane Buhamra Abreu

economia constitui-se em fator determinante para o fator 2. $\mathrm{O}$ fator 3 descreve medidas de economia. E o fator 4, medidas de reciclagem.

Tabela 7 - Cargas fatoriais do questionário de comportamentos ambientais

\begin{tabular}{ccccc}
\hline Questões & Fator 1 & Fator 2 & Fator 3 & Fator 4 \\
\hline Q26 & 0,132 & $\mathbf{- 0 , 5 5 1}$ & $-0,357$ & 0,117 \\
Q27 & 0,396 & $\mathbf{- 0 , 5 0 6}$ & 0,423 & 0,120 \\
Q28 & 0,429 & 0,098 & $\mathbf{- 0 , 4 6 2}$ & 0,482 \\
Q29 & $\mathbf{0 , 5 6 4}$ & $-0,427$ & 0,185 & 0,237 \\
Q30 & $\mathbf{0 , 6 8 7}$ & 0,215 & 0,321 & $-0,016$ \\
Q31 & $\mathbf{0 , 5 6 5}$ & 0,431 & 0,308 & $-0,094$ \\
Q32 & $\mathbf{0 , 4 7 4}$ & 0,345 & $-0,326$ & 0,201 \\
Q33 & $\mathbf{0 , 5 9 8}$ & 0,295 & $-0,180$ & 0,016 \\
Q34 & $\mathbf{0 , 5 1 8}$ & $-0,327$ & $-0,015$ & 0,031 \\
Q35 & 0,317 & $-0,239$ & $-0,417$ & $\mathbf{- 0 , 6 1 6}$ \\
Q36 & $\mathbf{0 , 5 5 1}$ & $-0,025$ & $-0,033$ & $-0,484$ \\
\hline \multicolumn{5}{c}{ Fonte: elaboração própria. }
\end{tabular}

A construção do modelo se deu em particionar o conjunto de dados de comportamentos ambientais nas seguintes escalas: medidas de economia explicado pelas afirmações 26,27,28 e 19; medidas de planejamento explicado pelas questões 30,31,32 e 33; medidas de reciclagem explicado pelas afirmações 34 e 35; e compra sustentável explicado pelas afirmações 36,37 e 38 , no entanto somente a questão 35 foi utilizada devido às questões 37 e 38 não estarem na mesma escala.

Como o teste Qui-quadrado é sensível ao tamanho da amostra, verificou-se a razão entre o valor assim obtido e os respectivos graus de liberdade. O valor resultante de 1,435 é considerado um bom ajuste.

Os índices de ajustamento também mostraram um bom ajuste do modelo para comportamento ambiental, como mostra Tabela 8.

Tabela 8 - Índices calculados para o modelo das escalas do comportamento ambiental

\begin{tabular}{cccccc}
\hline$\chi^{\mathbf{2}}$ (df) & valor $\mathbf{p}$ & CFI & RMSEA & RMSEA (IC) & SRMR \\
\hline $54,519(38)$ & 0,04 & 0,945 & 0,040 & $(0,009-0,063)$ & 0,052 \\
\hline \multicolumn{7}{c}{ Fonte: elaboração própria. }
\end{tabular}

A seguir, apresenta-se a análise descritiva da atitude e do comportamento sustentáveis dos estudantes de Administração participantes da pesquisa. 


\subsection{Análise descritiva da atitude sustentável}

A amostra foi composta de 138 (51,3\%) alunos do turno diurno e $131(48,7 \%)$ do noturno. Quanto ao semestre cursado, 48,4\% da amostra encontram-se entre o início e o meio do curso (do $1^{\circ}$ ao $5^{\circ}$ semestre) e $51,6 \%$, entre o meio e o final do curso (do $6^{\circ}$ ao $10^{\circ}$ semestre). A amostra ficou bem equilibrada entre alunos $(49,4 \%)$ e alunas $(50,6 \%)$, estando $80 \%$ dos respondentes entre 17 e 25 anos.

Abaixo, apresentam-se os resultados obtidos na pesquisa quanto à atitude sustentável dos alunos pesquisados. As frases relativas a cada afirmação apresentaram respostas padronizadas em escala Likert de 5 pontos, onde: (1) Discordo totalmente; (2) Discordo; (3) Nem concordo, nem discordo; (4) Concordo; e (5) Concordo totalmente. Para efeito de tabulação, as respostas 3 - não concordo nem discordo - foram consideradas negativas para atitudes e comportamentos pró-ambientais.

\subsubsection{Gravidade dos problemas ambientais}

A atitude dos respondentes quanto à gravidade dos problemas ambientais foi medida por 5 afirmativas (questões de 6 a 10), conforme demonstrado na Tabela 9.

Tabela 9 - Gravidade dos problemas ambientais

\begin{tabular}{c|c|c|c|c|c|c|c}
\hline \multirow{2}{*}{ Questões } & $\mathbf{7}$ Medidas descritivas \\
\cline { 2 - 8 } & $\mathbf{1}$ & $\mathbf{2}$ & $\mathbf{3}$ & $\mathbf{4}$ & $\mathbf{5}$ & Média & Desvio-Padrão \\
\hline Q6 & $85,87 \%$ & $11,90 \%$ & $1,49 \%$ & $0,74 \%$ & $0,00 \%$ & 1,17 & 0,46 \\
Q7 & $83,27 \%$ & $12,64 \%$ & $3,35 \%$ & $0,74 \%$ & $0,00 \%$ & 1,22 & 0,53 \\
Q8 & $84,76 \%$ & $12,27 \%$ & $1,49 \%$ & $1,49 \%$ & $0,00 \%$ & 1,20 & 0,53 \\
Q9 & $92,57 \%$ & $5,20 \%$ & $1,86 \%$ & $0,37 \%$ & $0,00 \%$ & 1,10 & 0,39 \\
Q10 & $86,25 \%$ & $8,92 \%$ & $3,35 \%$ & $0,74 \%$ & $0,74 \%$ & 1,21 & 0,60 \\
\hline \multicolumn{7}{c}{ Fonte: elaboração própria. }
\end{tabular}

Os resultados apresentados na Tabela 9 revelam que a grande maioria dos respondentes se preocupa com as questões ambientais, especialmente em relação à água (Q9), onde $97,77 \%$ da amostra discorda ou discorda totalmente da despreocupação com vazamentos e descargas. Vale destacar, entretanto, que embora em número muito pequeno, ainda há estudantes que não consideram necessário preocupar-se com reciclagem (Q6), com poluição (Q7), com economia de energia (Q8) e de água (Q9). 
Danielle Nunes Bezerra de Melo, Claudia Buhamra Abreu Romero, Hugo Osvaldo Acosta Reinado, Cristiane Buhamra Abreu

\subsubsection{Importância de ser ambientalmente correto}

A importância de ser ambientalmente correto foi avaliada por meio de três afirmativas relacionadas à reciclagem, como mostra a Tabela 10:

Tabela 10 - Importância de ser ambientalmente correto

\begin{tabular}{c|c|c|c|c|c|c|c}
\hline \multirow{2}{*}{ Questões } & \multicolumn{7}{c}{ Medidas descritivas } \\
\cline { 2 - 8 } & $\mathbf{1}$ & $\mathbf{2}$ & $\mathbf{3}$ & $\mathbf{4}$ & $\mathbf{5}$ & Média & Desvio-Padrão \\
\hline Q11 & $4,09 \%$ & $12,64 \%$ & $13,75 \%$ & $34,64 \%$ & $34,57 \%$ & 3,83 & 1,15 \\
Q12 & $0,37 \%$ & $2,60 \%$ & $5,20 \%$ & $29,37 \%$ & $62,45 \%$ & 4,51 & 0,74 \\
Q13 & $0,00 \%$ & $4,83 \%$ & $14,13 \%$ & $37,55 \%$ & $43,49 \%$ & 4,20 & 0,85 \\
\hline \multicolumn{7}{c}{ Fonte: elaboração própria. }
\end{tabular}

Os respondentes atribuíram grande importância às questões propostas, com média próxima a 4,0. Laroche et al. (2002) afirmam que a importância atribuída é uma atitude propícia para formar um comportamento ambientalmente correto. Destaque-se que apesar dos esforços de coleta seletiva empreendidos mundialmente, uma grande quantidade de respondentes não acredita que a reciclagem possa reduzir a poluição (Q11), que seja importante para preservar a natureza (Q12), nem que salvará terras usadas como depósitos de lixo (Q13).

\subsubsection{Nível de responsabilidade das corporações}

A avaliação do nível de responsabilidades das corporações foi medida por meio das questões 14 e 15. Na Tabela 7 observam-se as análises descritivas.

Tabela 11 - Nível de responsabilidade das corporações

\begin{tabular}{c|c|c|c|c|c|c|c}
\hline \multirow{2}{*}{ Questões } & \multicolumn{7}{c}{ Medidas descritivas } \\
\cline { 2 - 8 } & $\mathbf{1}$ & $\mathbf{2}$ & $\mathbf{3}$ & $\mathbf{4}$ & $\mathbf{5}$ & Média & Desvio-Padrão \\
\hline Q14 & $14,87 \%$ & $48,70 \%$ & $35,32 \%$ & $0,37 \%$ & $0,74 \%$ & 2,23 & 0,73 \\
Q15 & $8,55 \%$ & $41,64 \%$ & $38,66 \%$ & $10,78 \%$ & $0,37 \%$ & 2,53 & 0,81 \\
\hline \multicolumn{7}{c}{ Fonte: elaboração própria. }
\end{tabular}

O resultado obtido demonstrou que, entre os pesquisados, não há grande credibilidade em relação à responsabilidade ambiental empresarial, visto que a média obtida entre as duas questões foi de 2,38. Observa-se que para grande parte dos respondentes as corporações não estão cumprindo com sua responsabilidade ambiental uma vez que apenas $1,11 \%$ para o caso 
de empresas de alimentos embalados (Q14) e 11,15\% para empresas fabricantes de papel (Q15) concordam ou concordam totalmente.

Esse resultado demonstra que os esforços das corporações não estão sendo percebidos pelos estudantes. Como afirmam Peattie e Crane (2005), o greenwashing incita na sociedade uma desconfiança em relação à responsabilidade socioambiental das organizações (PEATTIE; CRANE, 2005).

\subsubsection{Inconveniência de ser ambientalmente correto}

Em relação à inconveniência de adotar uma postura sustentável, a maioria dos respondentes discorda que ser ambientalmente correto é inconveniente (Tabela 12).

Tabela 12 - Inconveniência de ser ambientalmente correto

\begin{tabular}{c|c|c|c|c|c|c|c}
\hline \multirow{2}{*}{ Questões } & \multicolumn{7}{c}{ Medidas descritivas } \\
\cline { 2 - 8 } & $\mathbf{1}$ & $\mathbf{2}$ & $\mathbf{3}$ & $\mathbf{4}$ & $\mathbf{5}$ & Média & Desvio-Padrão \\
\hline Q16 & $20,82 \%$ & $34,94 \%$ & $23,49 \%$ & $17,10 \%$ & $3,35 \%$ & 2,47 & 1,10 \\
Q17 & $73,61 \%$ & $22,68 \%$ & $2,23 \%$ & $1,12 \%$ & $0,37 \%$ & 1,32 & 0,61 \\
\hline \multicolumn{7}{c}{ Fonte: elaboração própria. }
\end{tabular}

Entretanto, ainda existe uma quantidade considerável de pessoas que acredita ser trabalhoso manter pilhas de lixo para reciclagem (Q16) e muitos não têm, sequer, opinião formada sobre isso, uma vez que nem concordam nem discordam. Surpreende ainda que cerca de, 4 pessoas $(1,49 \%)$ concordem que dá muito trabalho tentar controlar a poluição, por isso não vale a pena (Q17).

Segundo Laroche et al. (2002), a inconveniência é uma atitude relevante para determinar um comportamento. Mesmo que a importância dada pelo indivíduo a determinada questão seja alta, ele pode não adotar comportamentos condizentes por considerar o ato inconveniente.

\subsubsection{Pressão social}

As pressões sociais e legais são fatores importantes no estímulo ao comportamento pró-ambiental, aqui medidas por duas afirmativas, como mostra a Tabela 13. 
Tabela 13 - Pressão social

\begin{tabular}{c|c|c|c|c|c|c|c}
\hline \multirow{2}{*}{ Questões } & \multicolumn{7}{c}{ Medidas descritivas } \\
\cline { 2 - 8 } & $\mathbf{1}$ & $\mathbf{2}$ & $\mathbf{3}$ & $\mathbf{4}$ & $\mathbf{5}$ & Média & Desvio-Padrão \\
\hline Q18 & $30,11 \%$ & $41,26 \%$ & $17,10 \%$ & $7,06 \%$ & $4,46 \%$ & 2,14 & 1,07 \\
Q19 & $23,05 \%$ & $47,58 \%$ & $20,07 \%$ & $7,81 \%$ & $1,49 \%$ & 2,17 & 0,92 \\
\hline \multicolumn{7}{c}{ Fonte: elaboração própria. }
\end{tabular}

A maioria dos estudantes pesquisados afirmou não sentir as pressões sociais (Q18) nem as legais (Q19) em direção ao comportamento ambientalmente correto, com médias próximas a 2, como mostra a Tabela 13.

A seguir, são avaliados os comportamentos dos respondentes.

\subsection{Análise descritiva do comportamento sustentável}

O Teste do Consumo Consciente, desenvolvido pelo Instituto Akatu, organização não governamental sem fins lucrativos, "tem se revelado útil para compreender a evolução da consciência de consumo da população brasileira e mostrado resultados consistentes" (AKATU, 2013), desde 2006. O Teste é composto de 13 comportamentos sustentáveis, subdivididos em 4 categorias: Economia, Planejamento, Reciclagem e Compras sustentáveis. As respostas são dadas utilizando-se uma escala de quatro pontos: Nunca, Raramente, Às vezes e Sempre, e duas respostas dicotômicas: Sim ou Não.

A seguir, os resultados dos comportamentos dos respondentes são comparados com as atitudes apresentadas no bloco anterior.

\subsubsection{Medidas de economia}

Segundo o Instituto Akatu (2013), as medidas de economia "são práticas que resultam em benefícios diretos ao consumidor, causados pelo não desperdício de recursos, com retorno imediato e direto ao indivíduo praticante" (AKATU, 2013, p. 12). Os quatro comportamentos sustentáveis que representam economia estão na Tabela 14 (questões 20 a 23). 
Tabela 14 - Medidas de economia

\begin{tabular}{c|c|c|c|c|c|c}
\hline \multirow{2}{*}{ Questões } & \multicolumn{6}{|c}{ Medidas descritivas } \\
\cline { 2 - 7 } & Sempre & Às vezes & Raramente & Nunca & Média & Desvio-Padrão \\
\hline Q20 & $0,00 \%$ & $1,12 \%$ & $15,99 \%$ & $82,90 \%$ & 3,82 & 0,41 \\
Q21 & $0,37 \%$ & $2,97 \%$ & $33,83 \%$ & $62,83 \%$ & 3,59 & 0,57 \\
Q22 & $6,69 \%$ & $12,64 \%$ & $40,52 \%$ & $40,15 \%$ & 3,14 & 0,88 \\
Q23 & $2,23 \%$ & $8,92 \%$ & $45,35 \%$ & $43,49 \%$ & 3,30 & 0,72 \\
\hline
\end{tabular}

Fonte: elaboração própria.

O comportamento "costumo fechar a torneira quando escovo os dentes" (Q20) aparece como adotado sempre pela maioria dos respondentes. Esse resultado condiz com o elevado nível de preocupação com a água e a energia demonstrada na investigação sobre atitudes, e exposta na Tabela 9. Esse resultado confirma que atitudes com alto grau de confiança tendem a orientar comportamentos (BLACKWELL; MINIARD; ENGEL, 2008).

\subsubsection{Medidas de planejamento}

Para o Instituto Akatu (AKATU, 2013), as medidas de planejamento referem-se a "práticas que resultam no uso racional de recursos que, por serem planejadas antecipadamente, geram melhor aproveitamento e permitem um retorno individual a médio e longo prazos" (AKATU, 2013, p. 12). Os 4 comportamentos sustentáveis que representam Planejamento por parte do consumidor estão expostos na Tabela 15.

Tabela 15 - Medidas de planejamento

\begin{tabular}{c|c|c|c|c|c|c}
\hline \multirow{2}{*}{ Questões } & \multicolumn{6}{|c}{ Medidas descritivas } \\
\cline { 2 - 7 } & Sempre & Às vezes & Raramente & Nunca & Média & Desvio-Padrão \\
\hline Q24 & $8,92 \%$ & $18,59 \%$ & $36,06 \%$ & $36,43 \%$ & 3,00 & 0,95 \\
Q25 & $6,32 \%$ & $21,19 \%$ & $35,69 \%$ & $36,80 \%$ & 3,03 & 0,91 \\
Q26 & $10,78 \%$ & $20,45 \%$ & $41,26 \%$ & $27,51 \%$ & 2,86 & 0,94 \\
Q27 & $8,55 \%$ & $30,11 \%$ & $46,84 \%$ & $14,50 \%$ & 2,67 & 0,83 \\
\hline \multicolumn{7}{|c|}{ Fonte: elaboração própria. }
\end{tabular}

Surpreende constatar que Planejamento, uma das atividades primordiais de administradores, ainda não é uma prática adotada por muitos dos estudantes respondentes da pesquisa. Os comportamentos menos presentes entre os respondentes foram a leitura de rótulos no momento da compra (Q27) e a exigência da nota fiscal na compra (Q26). Considerando a atitude de pouca confiança dos respondentes em relação à postura ambiental das empresas (Tabela 11), era de se esperar maior cobrança a elas, o que não foi constatado. Pedir nota fiscal refere-se à perspectiva social do Triple Bottom Line (THE ECONOMIST, 
Danielle Nunes Bezerra de Melo, Claudia Buhamra Abreu Romero, Hugo Osvaldo Acosta Reinado, Cristiane Buhamra Abreu

2009), uma vez que sua emissão reflete o pagamento de impostos que possibilitarão serviços públicos à sociedade.

\subsubsection{Medidas de reciclagem}

Para o Instituto Akatu (AKATU, 2013), as medidas de reciclagem referem-se a "práticas diretamente ligadas ao descarte, reuso e reaproveitamento de materiais, em geral implicando, de modo mais significativo, em retorno para o coletivo da sociedade de médio ou longo prazo"(AKATU, 2013). Os dois comportamentos de Reciclagem estão expostos na Tabela 16.

Tabela 16 - Medidas de reciclagem

\begin{tabular}{c|c|c|c|c|c|c}
\hline \multirow{2}{*}{ Questões } & \multicolumn{6}{c}{ Medidas descritivas } \\
\cline { 2 - 7 } & Sempre & Ass vezes & Raramente & Nunca & Média & Desvio-Padrão \\
\hline Q28 & $1,49 \%$ & $1,86 \%$ & $30,48 \%$ & $66,17 \%$ & 3,61 & 0,60 \\
Q29 & $32,71 \%$ & $29,74 \%$ & $27,14 \%$ & $10,41 \%$ & 2,15 & 1,00 \\
\hline \multicolumn{6}{c}{ Fonte: elaboração própria. }
\end{tabular}

A utilização do verso das folhas de papel (Q28) é um comportamento presente na rotina de $66,17 \%$ dos respondentes. Apesar de ser a resposta da maioria dos respondentes, era de se esperar que esse percentual fosse ainda maior, dado que a atitude em relação à afirmativa "em nosso país há tantas árvores que não precisamos nos preocupar com reciclagem de papel" teve reprovação de 97,77\% (Q6 - Tabela 9). Outro comportamento que surpreende é a separação de lixo em casa (Q29) ser praticada por apenas $10,41 \%$ dos respondentes, e apresentando a média mais baixa entre todos os comportamentos $(2,15)$. Tal resultado não condiz com a atitude positiva apresentada pelos respondentes com relação à elevada importância atribuída à reciclagem (afirmativas com média total de 4,18 - Tabela 10), nem com o baixo nível de inconveniência atribuído à separação de lixo para a reciclagem (afirmativas com média total de 1,9 - Tabela 12). Esse gap entre atitude e comportamento pode ser justificado pela falta de pressão legal ou social, como apontado pelos respondentes nas questões 18 e 19 (Tabela 13), e pela falta de infraestrutura da cidade em relação à coleta seletiva sistematizada. 


\subsubsection{Medidas de compras sustentáveis}

Para o Instituto Akatu (2013), Compras Sustentáveis envolvem práticas que indicam a maior sensibilização do consumidor no plano do consumo consciente e sua mobilização diante das causas de sustentabilidade e responsabilidade social empresarial (AKATU, 2013). Os comportamentos de Compra Sustentável, medidos pelas questões 30,31 e 32, são expostos nas Tabelas 17 e 18.

Tabela 17 - Medidas de compras sustentáveis

\begin{tabular}{c|c|c|c|c|c|c}
\hline \multirow{2}{*}{ Questões } & \multicolumn{6}{|c}{ Medidas descritivas } \\
\cline { 2 - 7 } & Sempre & Às vezes & Raramente & Nunca & Média & Desvio-Padrão \\
\hline \multirow{2}{*}{ Q30 } & $5,20 \%$ & $25,28 \%$ & $47,21 \%$ & $22,30 \%$ & 2,87 & 0,82 \\
\hline
\end{tabular}

Fonte: elaboração própria.

Com a internet e as redes sociais dela oriundas, muitos são os compartilhamentos realizados $(\mathrm{Q} 30)$, haja vista o total de respostas às vezes e sempre que totalizam quase $70 \%$ dos respondentes expostas na Tabela 17.

Tabela 18 - Medidas de compras sustentáveis (respostas dicotômicas)

\begin{tabular}{c|c|c|c|c}
\hline \multirow{2}{*}{ Questões } & \multicolumn{4}{|c}{ Medidas descritivas } \\
\cline { 2 - 5 } & Não & Sim & Média & Desvio-Padrão \\
\hline Q31 & $41,64 \%$ & $58,36 \%$ & 1,58 & 0,49 \\
Q32 & $32,34 \%$ & $67,66 \%$ & 1,68 & 0,47 \\
\hline \multicolumn{4}{c}{ Fonte: elaboração própria. }
\end{tabular}

Os comportamentos de respostas dicotômicas - sim ou não - referem-se à compra de produtos orgânicos $(\mathrm{Q} 31)$ e de produtos reciclados (Q32), tendo ambos boa adesão pelos respondentes, como exposto na Tabela 18. Percebe-se, entretanto, que os produtos orgânicos ainda não são comprados por quase metade dos respondentes. A pesquisa Akatu 2012 (AKATU, 2013) ressalta o fato do "sustentável" ser vendido como mais um atributo de valor e, por isso, presumivelmente, relacionado a um preço mais alto. Embora, sob a ótica do "consumerismo consciente", indivíduos que se identificam com as questões ambientais desejem produtos sustentáveis e gastem dinheiro para tê-los (PEATTIE; CRANE, 2005; SOLOMON, 2011), o preço mais elevado pode fazer diferença na decisão de compra do público de estudantes aqui pesquisado. 
Danielle Nunes Bezerra de Melo, Claudia Buhamra Abreu Romero, Hugo Osvaldo Acosta Reinado, Cristiane Buhamra Abreu

\subsection{Avaliação do gap entre atitude e comportamento}

Considerando que a diferença de escalas para medir atitudes (de 5 pontos) e comportamentos (de 4 pontos) não permite realizar o Teste de Correlação, associaram-se as questões para avaliar estatisticamente se há gap entre ambos os tipos.

O teste de Qui-quadrado realizado mostra que houve diferença muito pequena, próxima a zero, para as questões Q21 - Evito deixar lâmpadas acesas em ambientes desocupados, e Q8 - Em países grandes como o Brasil, temos tanta eletricidade que não temos que nos preocupar com a economia de energia, isto é, com base na amostra, há evidências que existe associação entre essas duas questões. Houve também diferença muito pequena, próxima a zero, entre as questões 28 - Quando possível utilizo também o verso das folhas de papel, e Q6 - Em nosso país há tantas árvores que não precisamos nos preocupar com reciclagem de papel. Esse resultado mostra que para essas duas medidas de atitude e comportamento há evidência de associação.

Tabela 19 - Teste de associação entre as medidas de atitude e as de comportamento

\begin{tabular}{|c|c|}
\hline Questões & Valor p \\
\hline Q. 20 vs. Q. 9 & 0,213 \\
\hline Q. 21 vs. Q. 8 & $<0,001 *$ \\
\hline Q. 22 vs. Q. 8 & 0,715 \\
\hline Q. 23 vs. Q. 8 & 0,398 \\
\hline Q. 26 vs. Q. 14 & 0,696 \\
\hline Q. 26 vs. Q. 15 & 0,950 \\
\hline Q. 28 vs. Q. 6 & $<0,001 *$ \\
\hline Q. 29 vs. Q. 6 & 0,871 \\
\hline Q. 29 vs. Q. 7 & 0,110 \\
\hline Q. 29 vs. Q. 11 & 0,088 \\
\hline Q. 29 vs. Q. 13 & 0,970 \\
\hline Q. 29 vs. Q. 16 & 0,210 \\
\hline
\end{tabular}

Fonte: elaboração própria.

*Significativa ao nível de significância de 0,05 . 
Para as demais questões, os resultados mostram uma baixa associação entre elas, ou seja, as atitudes ambientais não estão associadas ao comportamento ambiental dos estudantes, indicando um gap entre o que pensam e sentem os estudantes pesquisados e a forma como agem em relação às questões de sustentabilidade.

\subsection{Segmentação dos respondentes quanto à quantidade de comportamentos adotados}

Seguindo o procedimento de pesquisa do Instituto Akatu (2013), a amostra foi segmentada de acordo com a adesão a comportamentos sustentáveis (Figura 1):

Figura 1 - Segmentação da amostra através dos comportamentos

Fonte: elaboração própria.

A segmentação baseia-se na quantidade de comportamentos que o respondente declara adotar sempre. Assim, são considerados conscientes os que responderam sempre praticar de 11 a 13 comportamentos; os engajados praticam sempre de 8 a 10 comportamentos; os Iniciantes, de 5 a 7 comportamentos, e os indiferentes de 0 a 4 comportamentos.

A pesquisa revela que uma parcela mínima de estudantes se classifica como consciente: apenas 3 estudantes. A maior parcela da amostra é considerada iniciante: 117 estudantes, e os engajados são 61. Merece destaque a constatação de que 88 estudantes são classificados como indiferentes.

Esse resultado confirma o gap existente entre a atitude e o comportamento ambiental dos respondentes. Laroche et al. (1996) explicam que muitos consumidores ainda não 
Danielle Nunes Bezerra de Melo, Claudia Buhamra Abreu Romero, Hugo Osvaldo Acosta Reinado, Cristiane Buhamra Abreu

possuem consciência do impacto que os seus hábitos de consumo acarretam nas questões ambientais e que uma boa parte de consumidores que expressam preocupação com o meio ambiente (atitude) não está disposta a assumir despesas pessoais ou mudanças no estilo de vida que adota (comportamentos).

O cruzamento dos resultados com as informações de gênero mostrou que as mulheres, assim como os mais velhos, acima de 35 anos, são mais sensíveis às questões de sustentabilidade. As variáveis turno e semestre não mostraram diferenças significativas.

\section{CONSIDERAÇÕES FINAIS}

O objetivo deste artigo foi investigar a atitude e o comportamento ambiental de futuros gestores organizacionais. Para tal, foram pesquisados 269 estudantes brasileiros do curso de Administração da FEAAC/UFC. A pesquisa de campo mostrou que, em relação às atitudes, os alunos mostraram-se preocupados com a gravidade dos problemas ambientais e consideram relevantes as atitudes ambientalmente corretas. O nível de responsabilidade das corporações foi considerado baixo, visto que os estudantes não depositam elevado grau de confiança nos comportamentos adotados pelas organizações, especialmente as fabricantes de alimentos embalados. Ainda sob a ótica das atitudes, o grau de inconveniência de ser ambientalmente correto, encontrado pela pesquisa, é baixo. As pressões sociais e legais sentidas pelos respondentes também foram consideradas baixas.

A análise dos comportamentos ligados ao consumo consciente mostra que a medida de economia é a mais adotada pelos estudantes, sendo o comportamento "costumo fechar a torneira quando escovo os dentes" o mais recorrente entre os 13 propostos. A medida de planejamento surge como a menos habitual entre os estudantes pesquisados, fato que merece atenção quando considerada a relevância que o planejamento possui para as atividades de profissionais de Administração.

As atitudes pró-ambientais dos estudantes de Administração da UFC são positivas, porém a maior parte dos participantes adota poucos comportamentos ambientais, sendo a maioria classificada como menos consciente. Preocupa o fato de apenas três estudantes estarem classificados como conscientes e de ainda existirem 88 indiferentes entre os 269 
pesquisados. A esperança é trazida pela grande maioria dos estudantes classificada como iniciantes e engajados.

Dos resultados da presente pesquisa, apreende-se a importância de incluir o tema da sustentabilidade na estrutura curricular dos cursos de Administração das instituições de ensino superior, o que já está sendo feito no curso da UFC. No entanto, a grande mudança não virá apenas com a inclusão de uma disciplina, mas com a abordagem do tema em todas as disciplinas curriculares, para que a formação dos futuros gestores seja completa e sustentável em todas as áreas da Administração.

Esta pesquisa teve como limitações investigar a realidade de estudantes de Administração de apenas uma universidade e selecionar a amostra com base em conveniência. Sugere-se, assim, para estudos futuros, aplicar a pesquisa a outras IES e até em outros cursos, de modo a garantir a formação de profissionais conscientes da importância de suas atitudes e comportamentos para a sustentabilidade das organizações e o bem-estar da humanidade.

\section{REFERÊNCIAS}

AJZEN, I. From Intentions to Actions: A Theory of Planned Behavior. In: KUHL, J.; BECKMANN, J. (Ed.) Action Control: from cognition to behavior, Berlin, Heidelberg, New York: Springer-Verlag, 1985, p. 11-39.

AKATU. Pesquisa Akatu 2012: rumo à sociedade do bem-estar. São Paulo, 2013. Disponível em: <https://www.akatu.org.br/wp-content/uploads/2017/04/PESQUISAAKATU.pdf>. Acesso em: 13 set. 2018.

BYRNE, B. M. Structural equation modeling with AMOS: Basic concepts, applications, and programming. Routledge, 2016. \{OU 2010?\}

CHARTER, M.; PEATTIE, K.; OTTMAN, J.; POLONSKY, M. Marketing and sustainability. Centre for Business Relationships, Accountability, Sustainability and Society (BRASS), in association with The Centre for Sustainable Design, 2002.

CRANE, A. Marketing and the Natural Environment: What Role for Morality? Journal of Macromarketing, v. 20, n. 2, 2000, p. 144-154. 
Danielle Nunes Bezerra de Melo, Claudia Buhamra Abreu Romero, Hugo Osvaldo Acosta Reinado, Cristiane Buhamra Abreu

DAFT, R. L. Administração. São Paulo: Cengage Learning, 2010.

DAHLSTROM, R. Gerenciamento de marketing verde. São Paulo: Cengage, 2011.

BLACKWELL, R. D.; MINIARD, P.; ENGEL, J. F. Comportamento do Consumidor. São Paulo: Cengage Learning, 9. ed, 2008.

FISHBEIN, M.; AJZEN I. Belief, attitude, intention and behavior: An introduction to theory and research. Reading, Massachusetts: AddisonWesley, 1975

FREITAS, E. J. X. Reflexão crítica e sustentabilidade da formação do administrador: o que revelam os discursos discentes? Dissertação (Mestrado em Administração de Empresas) Universidade Presbiteriana Mackenzie, São Paulo, 2014.

GIOVANNINI, F.; KRUGLIANSKAS, I. Fatores críticos de sucesso para a criação de um processo inovador sustentável de reciclagem: um estudo de caso. Revista de Administração Contemporânea, Curitiba, v. 12, n. 4, 2008, p. 931-951.

GOPALDAS, Ahir. Creating firm, customer, and societal value: Toward a theory of positive marketing. Journal of Business Research, 68, 2015, p. 2446-2451.

HAIR, Joseph F.; BLACK, William C.; BABIN, Barry J.; ANDERSON, Rolph E.; TATHAM, Ronald L. Análise multivariada de dados. Porto Alegre: Bookman, 2009.

INEP/MEC - Instituto Nacional de Estudos e Pesquisas Educacionais Anísio Teixeira, do Ministério da Educação. Inep divulga Indicadores de Qualidade da Educação Superior 2015, em 08 de março de 2017. Disponível em:

<http://portal.inep.gov.br/artigo/-/asset_publisher/B4AQV9zFY7Bv/content/id/666223>. Acesso em: 15/06/2017.

JACOBI, P. R.; RAULFFLET, E.; ARRUDA, M. P. Educação para a sustentabilidade nos cursos de administração: reflexão sobre paradigmas e práticas. RAM, Rev. Adm. Mackenzie, São Paulo, v. 12, n. 3, maio 2011, p. 21-50.

LAROCHE, M.; TOFFOLI, R.; KIM, C.; MULLER, T. The influence of culture on proenvironmental knowledge, attitudes, and behavior: a Canadian perspective. Advances in Consumer Research, 1996, p. 196-202. 
LAROCHE, M.; BERGERON, J.; TOMIUK, M.; BARBARO-FORLEO, G. Cultural differences in environmental knowledge, attitudes, and behaviors of Canadian consumers. Canadian Journal of Administrative Sciences, v. 18, n. 6, 2002, p. 267-282.

MOUTINHO, K.; ROAZZI, A. As teorias da ação racional e da ação planejada: relações entre intenções e comportamentos. Avaliação Psicológica. Porto Alegre: Springer Nature, v. 9, n. 2, ago. 2010, p. 279-287.

ONU - UNITED NATIONS. Report of the world commission on environment and development: our common future. 1987. Disponível em: <http://www.undocuments.net/wced-ocf.htm>. Acesso em: 20 fev. 2017.

OTTMAN, J. As novas regras do marketing verde: estratégias, ferramentas e inspiração para o branding sustentável. São Paulo: M. Books do Brasil, 2012.

PAUL, J.; MODI A.; PATEL, J. Predicting green product consumption using theory of planned behavior and reasoned action. Journal of Business Research, 29, 2016, p. 123-134.

PEATTIE, K..; CHARTER, M. Green Marketing. In: BAKER, M. J. (Ed.). The Marketing Book. 5. ed. Oxford: Elsevier, 2003. p. 726-755.

PEATTIE, K.; CRANE, A. Green marketing: legend, myth, farce or prophesy? Qualitative Market Research: An International Journal, v. 8, n. 4, 2005, p. 357-370.

PORTAL G1-CE. UFC é a segunda universidade mais procurada do país no Sisu 2018. Atualizado em 08/02/2018. Disponível em: <https://g1.globo.com/ce/ceara/noticia/universidade-federal-do-ceara-e-a-segunda-maisprocurada-do-brasil-no-sisu-2018.ghtml>. Acesso em: 09/02/2018.

ROMERO, C. B. A.; LAROCHE, M.; AURUP, G. M.; FERRAZ, S. B. Ethnicity and acculturation of environmental attitudes and behaviors: A cross-cultural study with Brazilians in Canada. Journal of Business Research, 82, 2017, p. 300-309.

RUF - Ranking Universitário Folha, 2017. Disponível em: <http://ruf.folha.uol.com.br/2017/ranking-de-universidades/>. Acesso em: 15/06/2018.

SOLOMON, M. R. O comportamento do consumidor: comprando, possuindo e sendo. 9. ed. Porto Alegre: Bookman, 2011. 
Danielle Nunes Bezerra de Melo, Claudia Buhamra Abreu Romero, Hugo Osvaldo Acosta Reinado, Cristiane Buhamra Abreu

THE ECONOMIST. Triple bottom line - It consists of three Ps: profit, people and planet. 17 nov. 2009. Disponível em: 〈http://www.economist.com/node/14301663>. Acesso em: 20 jul. 2015.

UNESCO. 2005-2014 - Década das Nações Unidas de Educação para o Desenvolvimento Sustentável. 2017. Disponível em: <http://www.unesco.org/new/pt/brasilia/about-thisoffice/prizes-and-celebrations/2005-2014-the-united-nations-decade-of-education-forsustainable-development/>. Acesso em: 28 maio 2017.

WU, Y. C.; HUANG, S.; KUO, L.; WU, W. H. Management Education for sustainability: a web-based content analysis. Academy Management Learning Education, v. 9, n. 3, p. 520$531,2010$. 